\title{
Boltzmann's Six-Moment One-Dimensional Nonlinear System Equations with the Maxwell-Auzhan Boundary Conditions
}

\author{
A. Sakabekov ${ }^{1}$ and Y. Auzhani ${ }^{2}$ \\ ${ }^{1}$ Research Laboratory “Applied Modeling Oil and Gas Fields”, Kazakh-British Technical University, 59 Tole Bi Street, \\ Almaty 050000, Kazakhstan \\ ${ }^{2}$ Al-Farabi Kazakh National University, 71 Al-Farabi Avenue, Almaty 050040, Kazakhstan \\ Correspondence should be addressed to A. Sakabekov; a.sakabekov@kbtu.kz
}

Received 2 February 2016; Revised 1 June 2016; Accepted 5 June 2016

Academic Editor: Peter G. L. Leach

Copyright (c) 2016 A. Sakabekov and Y. Auzhani. This is an open access article distributed under the Creative Commons Attribution License, which permits unrestricted use, distribution, and reproduction in any medium, provided the original work is properly cited.

We prove existence and uniqueness of the solution of the problem with initial and Maxwell-Auzhan boundary conditions for nonstationary nonlinear one-dimensional Boltzmann's six-moment system equations in space of functions continuous in time and summable in square by a spatial variable. In order to obtain a priori estimation of the initial and boundary value problem for nonstationary nonlinear one-dimensional Boltzmann's six-moment system equations we get the integral equality and then use the spherical representation of vector. Then we obtain the initial value problem for Riccati equation. We have managed to obtain a particular solution of this equation in an explicit form.

\section{Introduction}

In case of one-atom gas any macroscopic system during process of its evolution to an equilibrium state passes 3 stages: initial transition period, described in terms of a full function of system distribution; the kinetic period, by means of a onepartial distribution function; and the hydrodynamic period, by means of five first moments of the distribution function. In kinetic regime the behavior of a rarefied gas in space of time and velocity is described by Boltzmann's equation. It is known from gas dynamics that in most of the encountered problems there is no need to use detailed microscopic gas description with help of the distribution function. Therefore it is natural to look for a less detailed description using macroscopic hydrodynamic variables (density, hydrodynamic velocity, temperature, etc.). As these variables are defined in terms of moments of the distribution function, we are faced with the problem of analyzing the various moments of Boltzmann's equation.

Note that Boltzmann's moment equations are intermediate between Boltzmann (kinetic theory) and hydrodynamic levels of description of state of the rarefied gas and form class of nonlinear partial differential equations. Existence of such class of equations was noticed by Grad [1, 2] in 1949 .
He obtained the moment system by expanding the particle distribution function in Hermite polynomials near the local Maxwell distribution. Grad used Cartesian coordinates of velocities and Grad's moment system contained as coefficients such unknown hydrodynamic characteristics like density, temperature, average speed, and so forth. Formulation of boundary conditions for Grad's system is almost impossible, as the characteristic equations for various approximations of Grad's hyperbolic system contain unknown parameters like density, temperature, and average speed. However, 13- and 20 -moment Grad equations are widely used in solving many problems of the kinetic theory of gases and plasma.

Boltzmann's equation is equivalent to an infinite system of differential equations relative to the moments of the particle distribution function in the complete system of eigenfunctions of linearized operator. As a rule we limit study by finite moment system of equations because solving infinite system of equations does not seem to be possible.

Finite system of moment equations for a specific task with a certain degree of accuracy replaces Boltzmann's equation. It is necessary, also roughly, to replace the boundary conditions for the particle distribution function by a number of macroscopic conditions for the moments; that is, there arises the problem of boundary conditions for a finite system of 
equations that approximate the microscopic boundary conditions for Boltzmann's equation. The question of boundary conditions for a finite system of moment equations can be divided into two parts: how many conditions must be imposed and how they should be prepared. From microscopic boundary conditions for Boltzmann's equation there can be obtained an infinite set of boundary conditions for each type of decomposition. However, the number of boundary conditions is determined not by the number of moment equations; that is, it is impossible, for example, to take as many boundary conditions as equations, although the number of moment equations affects the number of boundary conditions. In addition, the boundary conditions must be consistent with the moment equations and the resulting problem must be correct. Boundary condition problems arise in the following tasks: (1) moment boundary conditions in rarefied gas slip-flow problems; (2) definition of boundary conditions on the surfaces of streamlined rarefied gas; (3) prediction aerospace aerodynamic characteristics of aircraft at very high speeds and at high altitudes and so forth.

In work [3] we have obtained the moment system which differs from Grad's system of equations. We used spherical velocity coordinates and decomposed the distribution function into a series of eigenfunctions of the linearized collision operator $[4,5]$, which is the product of Sonine polynomials and spherical functions. The resulting system of equations, which correspond to the partial sum of series and which we called Boltzmann's moment system of equations, is a nonlinear hyperbolic system in relation to the moments of the particles distribution function.

The structure of Boltzmann's moment system of equations corresponds to the structure of Boltzmann's equation; namely, the differential part of the resulting system is linear in relation to the moments of the distribution function and nonlinearity is included as moments of collision integral [6].

The linearity of differential part of Boltzmann's moment system of equations simplifies the task of formulation of the boundary conditions. In work [3] a homogeneous boundary condition for particles distribution function was approximated and proved the correctness of initial and boundary value problem for nonlinear nonstationary Boltzmann's moment system of equations in three-dimensional space. In work [7] the initial and boundary value problem for one-dimensional nonstationary Boltzmann's equation with boundary conditions of Maxwell was approximated by a corresponding problem for Boltzmann's moment system of equations. The boundary conditions for Boltzmann's moment system of equations were called Maxwell-Auzhan conditions.

In work [8] a systematic nonperturbative derivation of a hierarchy of closed systems of moment equations corresponding to any classical theory has been presented. This paper is a fundamental work where closed systems of moment equations describe a transition regime. Moreover, hydrodynamical model is used to describe charge transport in a generic compound semiconductor. Compound semiconductors have found wide use in the microelectronic industry. The evolution equation for macroscopic variables is obtained by taking moments of the transport equation [913].
The study of various problems for Boltzmann's moment system of equations is an important and actual task in the theory of a rarefied gas and other applications of the moment system equations. The correctness of initial and boundary problems for Boltzmann's moment system of equations with Maxwell-Auzhan boundary conditions is being studied for the first time.

\section{Existence and Uniqueness of the Solutions of Initial and Boundary Value Problem for Six-Moment One-Dimensional Boltzmann's System of Equations with Maxwell-Auzhan Boundary Conditions}

In this section we prove the existence and uniqueness of solutions of the initial and boundary value problem for six-moment one-dimensional Boltzmann's system of equations with Maxwell-Auzhan boundary conditions in space of functions, continuous in time and summable in square by spatial variable. Note that the theorem of the existence of a global solution in time of the initial and boundary value problem for 3-dimensional nonlinear Boltzmann's equation with boundary conditions of Maxwell is proved in work [14].

We write in an expanded form system of one-dimensional Boltzmann's moment equations in the $k$ th approximation, which corresponds to decomposition of the particle distribution function by eigenfunctions of the linearized collision operator

$$
\begin{aligned}
& \frac{\partial f_{n l}}{\partial t}+\frac{1}{\alpha} \frac{\partial}{\partial x}\left[l \left(\sqrt{\frac{2(n+l+1 / 2)}{(2 l-1)(2 l+1)}} f_{n, l-1}\right.\right. \\
& \left.\quad-\sqrt{\frac{2(n+1)}{(2 l-1)(2 l+1)}} f_{n+1, l-1}\right)+(l+1) \\
& \\
& \quad\left(\sqrt{\frac{2(n+l+1 / 2)}{(2 l+1)(2 l+3)}} f_{n, l+1}\right. \\
& \left.\left.-\sqrt{\frac{2 n}{(2 l+1)(2 l+3)}} f_{n-1, l+1}\right)\right]=I_{n l}, \\
& 2 n+l=0,1, \ldots, k,
\end{aligned}
$$

where the moments $I_{n l}$ of a nonlinear collision operator are expressed through coefficients of Talmi and Klebsh-Gordon $[15,16]$ and have the form

$$
\begin{aligned}
I_{n l} & =\sum\left\langle N_{3} L_{3} n_{3} l_{3}: l \mid n l 00: l\right\rangle \\
& \cdot\left\langle N_{3} L_{3} n_{3} l_{3}: l \mid n_{1} l_{1} n_{2} l_{2}: l\right\rangle\left(\frac{l_{1} 0 l_{2} 0}{l 0}\right)\left(\sigma_{l_{3}}-\sigma_{0}\right) \\
& \cdot f_{n_{1} l_{1}} f_{n_{2} l_{2}},
\end{aligned}
$$

where $\left\langle N_{3} L_{3} n_{3} l_{3}: l \mid n_{1} l_{1} n_{2} l_{2}: l\right\rangle$ are generalized coefficients of Talmi, $\left(l_{1} 0 l_{2} 0 / l 0\right)$ are Klebsh-Gordon coefficients, $\alpha=$ $1 / \sqrt{R \theta}$ is the constant, $R$ is Boltzmann's constant, and $\theta$ is the ideal gas temperature. 
For generalized coefficients of Talmi there exists a table [15] for each value of quantum number $\xi=2 n+l$ from 0 to 6 . Moreover, there is a program on IBM for calculation of generalized coefficients of Talmi.

If in (1) $2 n+l$ takes values from 0 to 3 , we get Boltzmann's moment system equations in the third approximation. We write it in an expanded form:

$$
\begin{aligned}
& \frac{\partial f_{00}}{\partial t}+\frac{1}{\alpha} \frac{\partial f_{01}}{\partial x}=0 \\
& \frac{\partial f_{02}}{\partial t}+\frac{1}{\alpha} \frac{\partial}{\partial x}\left(\frac{2}{\sqrt{3}} f_{01}+\frac{3}{\sqrt{5}} f_{03}-\frac{2 \sqrt{2}}{\sqrt{15}} f_{11}\right)=J_{02} \\
& \frac{\partial f_{10}}{\partial t}+\frac{1}{\alpha} \frac{\partial}{\partial x}\left(-\sqrt{\frac{2}{3}} f_{01}+\sqrt{\frac{5}{3}} f_{11}\right)=0 \\
& \frac{\partial f_{01}}{\partial t}+\frac{1}{\alpha} \frac{\partial}{\partial x}\left(f_{00}+\frac{2}{\sqrt{3}} f_{02}-\sqrt{\frac{2}{3}} f_{10}\right)=0 \\
& \frac{\partial f_{03}}{\partial t}+\frac{1}{\alpha} \frac{\partial}{\partial x} \frac{3}{\sqrt{5}} f_{02}=J_{03}, \\
& \frac{\partial f_{11}}{\partial t}+\frac{1}{\alpha} \frac{\partial}{\partial x}\left(-\frac{2 \sqrt{2}}{\sqrt{15}} f_{02}+\sqrt{\frac{5}{3}} f_{10}\right)=J_{11}, \\
& x \in(-a, a), t>0
\end{aligned}
$$

where $f_{00}=f_{00}(t, x)$ and $f_{01}=f_{01}(t, x), \ldots, f_{11}=f_{11}(t, x)$ are the moments of the particle distribution function; $J_{02}=$ $\left(\sigma_{2}-\sigma_{0}\right)\left(f_{00} f_{02}-f_{01}^{2} / \sqrt{3}\right) / 2, J_{03}=(1 / 4)\left(\sigma_{3}+3 \sigma_{1}-\right.$ $\left.4 \sigma_{0}\right) f_{00} f_{03}+(1 / 4 \sqrt{5})\left(2 \sigma_{1}+\sigma_{0}-3 \sigma_{3}\right) f_{01} f_{02}$, and $J_{11}=$ $\left(\sigma_{1}-\sigma_{0}\right)\left(f_{00} f_{11}+(1 / 2) \sqrt{5 / 3} f_{10} f_{01}-(\sqrt{2} / \sqrt{15}) f_{01} f_{02}\right)$ are moments of the collision integral, where $\sigma_{0}, \sigma_{1}, \sigma_{2}$, and $\sigma_{3}$ are the Fourier coefficients of the cross section expansion by the Legendre polynomials. The first, third, and fourth equations of system (3) correspond to mass conservation law, momentum conservation law, and energy conservation law, respectively.

We study the correctness of initial and boundary value problem for six-moment one-dimensional Boltzmann's system equations

$$
\begin{aligned}
\frac{\partial u}{\partial t}+A \frac{\partial w}{\partial x} & =J_{1}(u, w) \\
\frac{\partial w}{\partial t}+A^{\prime} \frac{\partial u}{\partial x} & =J_{2}(u, w), \\
\left.u\right|_{t=0} & =u_{0}(x), \\
\left.w\right|_{t=0} & =w_{0}(x),
\end{aligned}
$$

$$
\begin{aligned}
\left.\left(A w^{-}+B u^{-}\right)\right|_{x=-a}= & \left.\frac{1}{\beta}\left(A w^{+}-B u^{+}\right)\right|_{x=-a} \\
& -\frac{(1-\beta) \eta}{\alpha \beta \sqrt{\pi}} F, \quad t \in[0, T], \\
\left.\left(A w^{-}-B u^{-}\right)\right|_{x=a}= & \left.\frac{1}{\beta}\left(A w^{+}+B u^{+}\right)\right|_{x=a} \\
& +\frac{(1-\beta) \eta}{\alpha \beta \sqrt{\pi}} F, \quad t \in[0, T],
\end{aligned}
$$

where

$$
\begin{aligned}
& A=\frac{1}{\alpha}\left(\begin{array}{ccc}
1 & 0 & 0 \\
\frac{2}{\sqrt{3}} & \frac{3}{\sqrt{5}} & -\frac{2 \sqrt{2}}{\sqrt{15}} \\
-\sqrt{\frac{2}{3}} & 0 & \sqrt{\frac{5}{3}}
\end{array}\right), \\
& B=\frac{1}{\alpha \sqrt{\pi}}\left(\begin{array}{ccc}
\sqrt{2} & \sqrt{\frac{2}{3}} & -\frac{1}{\sqrt{3}} \\
\sqrt{\frac{2}{3}} & 2 \sqrt{2} & -1 \\
-\frac{1}{\sqrt{3}} & -1 & 3 \sqrt{2}
\end{array}\right) \\
& J_{1}(u, w)=\left(0, J_{02}, 0\right)^{\prime}, \\
& J_{2}(u, w)=\left(0, J_{03}, J_{11}\right)^{\prime}, \\
& u=\left(f_{00}, f_{02}, f_{10}\right)^{\prime}, w=\left(f_{01}, f_{03}, f_{11}\right)^{\prime}, \\
&\left.\frac{1}{4 \sqrt{2}}, \frac{1}{8 \sqrt{6}}, \frac{1}{8 \sqrt{3}}\right)^{\prime} .
\end{aligned}
$$

$A^{\prime}$ is the transpose matrix; $B$ is the positive definite matrix; $u_{0}(x)=\left(f_{00}^{0}(x), f_{02}^{0}(x), f_{10}^{0}(x)\right)^{\prime}$ and $w_{0}(x)=$ $\left(f_{01}^{0}(x), f_{03}^{0}(x), f_{11}^{0}(x)\right)^{\prime}$ are given initial vector-functions; $w^{+}, u^{+}$are vector moments of falling to the boundary particle distribution function; $w^{-}, u^{-}$are vector moments of reflecting from the boundary particle distribution function. Equation (4) is vector matrix form of system equations (3).

It is possible to check through direct calculations that

$$
\operatorname{det} A_{1}=\operatorname{det}\left(\begin{array}{cc}
0 & A \\
A^{\prime} & 0
\end{array}\right) \neq 0 \text {, }
$$

and matrix $A_{1}$ has three positive and the same number of negative nonzero eigenvalues. From (6)-(7) it follows that the number of boundary conditions on the left and right ends of interval $(-a, a)$ is equal to the number of positive and negative eigenvalues of the matrix $A_{1}$.

Thus system (4) is a symmetric hyperbolic nonlinear partial differential equations system. Let us show that $J_{02}$ 
is a sign-nondefined square form. It is easy to check that $f_{00} f_{02}-\left(f_{01}^{2} / \sqrt{3}\right)=(C U, U)$, where $U=(u, w)^{\prime}$ and

$$
C=\left(\begin{array}{cccccc}
0 & \frac{1}{2} & 0 & 0 & 0 & 0 \\
\frac{1}{2} & 0 & 0 & 0 & 0 & 0 \\
0 & 0 & 0 & 0 & 0 & 0 \\
0 & 0 & 0 & -\frac{1}{\sqrt{3}} & 0 & 0 \\
0 & 0 & 0 & 0 & 0 & 0 \\
0 & 0 & 0 & 0 & 0 & 0
\end{array}\right) .
$$

Eigenvalues of matrix $C$ are $-1 / 2,-1 / \sqrt{3}, 0,0,0$, and $1 / 2$. Therefore $J_{02}$ is a sign-nondefined square form. Similarly, we can show that $J_{03}$ and $J_{11}$ are also sign-nondefined square forms.

For problem (4)-(7) the following theorem takes place.

Theorem 1. If $U_{0}=\left(u_{0}(x), w_{0}(x)\right) \in L^{2}[-a, a]$, then problem (4)-(7) has a unique solution in domain $[-a, a] \times[0, T]$ belonging to the space $C\left([0, T] ; L^{2}[-a, a]\right)$; moreover

$$
\|\| U\left\|_{L^{2}[-a, a]}-r_{1}\right\|_{C[0, T]} \leq C_{1}\left(\left\|U_{0}\right\|_{L^{2}[-a, a]}-r_{1}(0)\right),
$$

where $C_{1}$ is a constant independent from $U$ and $T \sim$ $\left.\mathrm{O}\left(\left\|U_{0}\right\|_{L^{2}[-a, a]}-r_{1}(0)\right)^{-1}\right), r_{1}(t)$ is the particular solution of Riccati equation (15) in an explicit form.

Proof. Let $U_{0} \in L^{2}[-a, a]$. Let us prove estimation (11). We multiply the first equation in system (4) by $u$ and the second equation by $w$ and perform integration from $-a$ to $a$ :

$$
\begin{aligned}
& \frac{1}{2} \frac{d}{d t} \int_{-a}^{a}[(u, u)+(w, w)] d x \\
& \quad+\int_{-a}^{a}\left[\left(A \frac{\partial w}{\partial x}, u\right)+\left(A^{\prime} \frac{\partial u}{\partial x}, w\right)\right] d x \\
& \quad=\int_{-a}^{a}\left[\left(J_{1}, u\right)+\left(J_{2}, w\right)\right] d x .
\end{aligned}
$$

After integration by parts we get

$$
\begin{aligned}
& \frac{1}{2} \frac{d}{d t} \int_{-a}^{a}[(u, u)+(w, w)] d x+\left.\left(u^{-}, A w^{-}\right)\right|_{x=a} \\
& -\left.\left(u^{-}, A w^{-}\right)\right|_{x=-a}=\int_{-a}^{a}\left[\left(J_{1}, u\right)+\left(J_{2}, w\right)\right] d x .
\end{aligned}
$$

Taking into account boundary conditions (6)-(7) we rewrite equality (13) in the following form:

$$
\begin{aligned}
& \frac{1}{2} \frac{d}{d t} \int_{-a}^{a}[(u, u)+(w, w)] d x+\left.\left(B u^{-}, u^{-}\right)\right|_{x=a} \\
& \quad+\left.\left(B u^{-}, u^{-}\right)\right|_{x=-a}-\left.\frac{1}{\beta}\left(\left(A w^{+}-B u^{+}\right), u^{-}\right)\right|_{x=-a} \\
& \quad+\left.\frac{1}{\beta}\left(\left(A w^{+}+B u^{+}\right), u^{-}\right)\right|_{x=a}+\left.\left(F_{1}, u^{-}\right)\right|_{x=a}
\end{aligned}
$$

$$
\begin{aligned}
& +\left.\left(F_{1}, u^{-}\right)\right|_{x=-a} \\
= & \int_{-a}^{a}\left[\left(J_{1}(u, w), u\right)+\left(J_{2}(u, w), w\right)\right] d x
\end{aligned}
$$

where $F_{1}=((1-\beta) \eta / \alpha \beta \sqrt{\pi}) F$.

Let us use the spherical representation [17] of vector $U(t, x)=r(t) \omega(t, x)$, where $\omega(t, x)=\left(\omega_{1}(t, x), \omega_{2}(t, x)\right)^{\prime}$, $r(t)=\|U(t, \cdot)\|_{L^{2}[-a, a]}$, and $\|\omega\|_{L^{2}[-a, a]}=1$. Furthermore assume that $\omega(t, x) \in C^{2}[0, T]$, where the value of $T$ we define below.

Substituting the values $u=r(t) \omega_{1}(t, x)$ and $w=$ $r(t) \omega_{2}(t, x)$ into (14) we have that

$$
\frac{d r}{d t}+r P(t)=r^{2} Q(t)-f(t)
$$

where

$$
\begin{aligned}
& P(t)=\left.\left(B \omega_{1}^{-}, \omega_{1}^{-}\right)\right|_{x=a}+\left.\left(B \omega_{1}^{-}, \omega_{1}^{-}\right)\right|_{x=-a} \\
& +\frac{1}{\beta}\left[\left.\left(A \omega_{2}^{+}, \omega_{1}^{-}\right)\right|_{x=a}+\left.\left(B \omega_{1}^{+}, \omega_{1}^{-}\right)\right|_{x=a}\right. \\
& \left.\quad+\left.\left(B \omega_{1}^{+}, \omega_{1}^{-}\right)\right|_{x=-a}-\left.\left(A \omega_{2}^{+}, \omega_{1}^{-}\right)\right|_{x=-a}\right], \\
& Q(t)=\int_{-a}^{a}\left[\left(J_{1}\left(\omega_{1}, \omega_{2}\right), \omega_{1}\right)+\left(J_{2}\left(\omega_{1}, \omega_{2}\right), \omega_{2}\right)\right] d x, \\
& f(t)=\left.\left(F_{1}, \omega_{1}^{-}\right)\right|_{x=a}+\left.\left(F_{1}, \omega_{1}^{-}\right)\right|_{x=-a} .
\end{aligned}
$$

Let us study (15) with an initial condition

$$
r(0)=\left\|U_{0}\right\|=\left\|U_{0}\right\|_{L^{2}[-a, a]} .
$$

Let $r_{1}(t)$ be a particular solution of Riccati equation (15). Then the general solution of (15) has the form

$$
\begin{aligned}
& r(t)=r_{1}(t)+\exp \left(\int_{0}^{t}\left(2 Q(\tau) r_{1}(\tau)-P(\tau)\right) d \tau\right)[C \\
& -\int_{0}^{t} Q(\tau) \\
& \left.\quad \cdot \exp \left(\int_{0}^{\tau}\left(2 Q(\xi) r_{1}(\xi)-P(\xi)\right) d \xi\right) d \tau\right]^{-1} \cdot
\end{aligned}
$$

Hence, taking into consideration initial condition (17) we obtain 


$$
r(t)=r_{1}(t)+\exp \left(\int_{0}^{t}\left(2 Q(\tau) r_{1}(\tau)-P(\tau)\right) d \tau\right)\left[\frac{1}{\left\|U_{0}\right\|-r_{1}(0)}-\int_{0}^{t} Q(\tau) \exp \left(\int_{0}^{\tau}\left(2 Q(\xi) r_{1}(\xi)-P(\xi)\right) d \xi\right) d \tau\right]^{-1}
$$

If $R(t) \equiv \int_{0}^{t} Q(\tau) \exp \left(\int_{0}^{\tau}\left(2 Q(\xi) r_{1}(\xi)-P(\xi)\right) d \xi\right) d \tau \leq 0 \forall t$, then the second term of $r(t)$ is bounded $\forall t \in[0,+\infty)$ under the condition that difference $\left\|U_{0}\right\|-r_{1}(0)$ is positive. Further we suppose a difference $\left\|U_{0}\right\|-r_{1}(0)$ is a positive number. Let $R(t)>0$. We denote by $T_{1}$ the moment of time at which

$$
\begin{aligned}
& \frac{1}{\left\|U_{0}\right\|-r_{1}(0)} \\
& \quad-\int_{0}^{T_{1}} Q(\tau) \exp \left(\int_{0}^{\tau}\left(2 Q(\xi) r_{1}(\xi)-P(\xi)\right) d \xi\right) d \tau \\
& =0 .
\end{aligned}
$$

Then $r(t)-r_{1}(t)$ is bounded $\forall t \in[0, T]$, where $T<T_{1}$ and $T_{1} \sim \mathrm{O}\left(1 /\left(\left\|U_{0}\right\|-r_{1}(0)\right)\right)$. Analyzing structure of $r_{1}(t)$ (see (31)) from equality (20) we obtain $Q(t)>0$ for any $t \in[0, T]$.

Now we found one particular solution of (15). The nonlinear Riccati equation can always be reduced to a secondorder linear ordinary differential equation [18]

$$
y^{\prime \prime}+f_{1}(t) y^{\prime}+f_{2}(t) y=0
$$

where

$$
\begin{aligned}
y(t) & =\exp \left(-\int_{0}^{t} Q(\tau) r(\tau) d \tau\right), \\
f_{1}(t) & =\frac{Q(t) P(t)-Q^{\prime}(t)}{Q(t)}, \\
f_{2}(t) & =Q(t) f(t) .
\end{aligned}
$$

Solution of this equation will lead to a solution of $r(t)=$ $-y^{\prime} / y \mathrm{Q}(t)$ of the original Riccati equation. By substitution

$$
y(t)=z(t) \exp \left(-\frac{1}{2} \int_{0}^{t} f_{1}(\tau) d \tau\right)
$$

we write (21) in the following form [18]:

$$
z^{\prime \prime}(t)+a(t) z(t)=0,
$$

where $a(t)=f_{2}(t)-(1 / 2) f_{1}^{\prime}(t)-(1 / 4) f_{1}^{2}(t)$.

If instead of $f_{1}(t)$ and $f_{2}(t)$ we substitute values from (22), then we get

$$
\begin{aligned}
a(t)= & Q f(t) \\
& -\frac{2\left(Q^{2} P^{\prime}-Q^{\prime \prime} Q+Q^{\prime 2}\right)+\left(Q P-Q^{\prime}\right)^{2}}{4 Q^{2}} .
\end{aligned}
$$

In work [19] it was proven that function

$$
z(t)=1+\sum_{k=1}^{\infty} b_{k}(t)
$$

is the solution of (24), where

$$
\begin{aligned}
& b_{1}(t)=-\int_{0}^{t} \int_{0}^{\xi} a(\tau) d \tau d \xi \\
& b_{k}(t)=-\int_{0}^{t} \int_{0}^{\xi} a(\tau) b_{k-1}(\tau) d \tau d \xi
\end{aligned}
$$

and the series on the right hand side of (26) uniformly converge.

Then

$$
\begin{aligned}
y & =\left(1+\sum_{k=1}^{\infty} b_{k}(t)\right) \exp \left(-\frac{1}{2} \int_{0}^{t} f_{1}(\tau) d \tau\right), \\
r_{1}(t) & =-\frac{y^{\prime}}{y Q}=-\frac{z^{\prime}(t)-(1 / 2) z(t) f_{1}(t)}{z(t) Q(t)}
\end{aligned}
$$

is a particular solution of Riccati equation (15).

Since $z^{\prime}(0)=0$, value of

$$
r_{1}(0)=-\frac{z^{\prime}(0)-(1 / 2) z(0) f_{1}(0)}{z(0) Q(0)}=\frac{f_{1}(0)}{2 Q(0)} .
$$

Now instead of $f_{1}(0)$ we substitute its value $P(0)$ $Q^{\prime}(0) / Q(0)$ and get $r_{1}(0)=\left(P(0) Q(0)-Q^{\prime}(0)\right) / 2 Q^{2}(0)$.

The value of $Q(t)$ with $t=0$ is

$Q(0)$

$=\left.\int_{-a}^{a}\left(\left(J_{1}\left(\omega_{1}, \omega_{2}\right), \omega_{1}\right)+\left(J_{2}\left(\omega_{1}, \omega_{2}\right), \omega_{2}\right)\right)\right|_{t=0} d x$

$>0$,

since $Q(t)>0$ for any $t \in[0, T]$.

An expansion form of the function

$$
\begin{gathered}
r_{1}(t)=\frac{1}{Q(t)}\left[\frac{1}{z(t)} \int_{0}^{t} a(\tau) z(\tau) d \tau\right. \\
\left.+\frac{Q(t) P(t)-Q^{\prime}(t)}{2 Q(t)}\right] .
\end{gathered}
$$

It is not difficult to prove using immediate substitution that function $r_{1}(t)$ satisfies Riccati equation (15).

From equality (19) we obtain

$$
\left|r(t)-r_{1}(t)\right| \leq C\left(\left\|U_{0}\right\|-r_{1}(0)\right) .
$$

Hence

$$
\begin{gathered}
\left|\|U(t, \cdot)\|_{L^{2}[-a, a]}-r_{1}(t)\right| \leq C\left(\left\|U_{0}\right\|-r_{1}(0)\right), \\
\|\| U\left\|_{L^{2}[-a, a]}-r_{1}\right\|_{C[0, T]} \leq C\left(\left\|U_{0}\right\|-r_{1}(0)\right) .
\end{gathered}
$$


We take as interval of the solution of problem (15)(17) segment $\left[0 ; 1 /\left(\left\|U_{0}\right\|-r_{1}(0)\right)\right]$, since the integrand $Q(\tau) \exp \left(\int_{0}^{\tau}\left(2 Q(\xi) r_{1}(\xi)-P(\xi)\right) d \xi\right)$ is bounded. Hence $\forall t \in$ $[0, T]$ (where $T \approx T_{1}$ ) a priori estimation (11) takes place.

Now we prove the existence of solution for (4)-(7) using Galerkin method. Let $\left\{v_{l}(x)\right\}_{l=1}^{\infty}$ be a basis in space $L^{2}[-a, a]$, where the dimension of vector $v_{l}(x)$ is equal to the dimension of vector $U$. For each $m$ we define an approximate solution $U_{m}$ of (4)-(7) as follows:

$$
\begin{aligned}
& U_{m}=\sum_{j=1}^{m} c_{j m}(t) v_{j}(x), \\
& \int_{-a}^{a}\left(\left(\frac{\partial U_{m}}{\partial t}+A_{1} \frac{\partial U_{m}}{\partial x}\right), v_{i}(x)\right) d x \\
& =\int_{-a}^{a}\left(J\left(U_{m}\right), v_{i}(x)\right) d x, \quad i=\overline{1, m}, t \in(0, T], \\
& \left.U_{m}\right|_{t=0}=U_{0 m}(x), \quad x \in R, \\
& \left.\left(A w_{m}^{-} \mp B u_{m}^{-}\right)\right|_{x=\mp a} \\
& =\left.\frac{1}{\beta}\left(A w_{m}^{+} \pm B u_{m}^{+}\right)\right|_{x= \pm a} \pm \frac{(1-\beta) \eta}{\alpha \beta \sqrt{\pi}} F
\end{aligned}
$$

where $U_{0 m}$ is the orthogonal projection in $L^{2}$ of the function $U_{0}$ on the subspace, spanned by $v_{1}, \ldots, v_{m}$,

$$
J\left(U_{m}\right)=\left(J_{1}\left(u_{m}, w_{m}\right), J_{2}\left(u_{m}, w_{m}\right)\right)^{\prime} .
$$

We represent $v_{j}(x)$ in form $v_{j}(x)=\left(v_{j}^{(1)}, v_{j}^{(2)}\right)^{\prime}$, where

$$
\begin{aligned}
& v_{j}^{(1)}=\left(v_{j 1}, v_{j 2}, v_{j 3}\right)^{\prime}, \\
& v_{j}^{(2)}=\left(v_{j 4}, v_{j 5}, v_{j 6}\right)^{\prime} .
\end{aligned}
$$

The coefficients $c_{j m}(t)$ are determined from the equations

$$
\begin{aligned}
\sum_{j=1}^{m} & \left\{\frac{d c_{j m}}{d t} \int_{-a}^{a}\left(v_{j}, v_{i}\right) d x+c_{j m}\left[\left.\left(B v_{j}^{-(1)}, v_{i}^{-(1)}\right)\right|_{x=a}\right.\right. \\
& +\left.\left(B v_{j}^{-(1)}, v_{i}^{-(1)}\right)\right|_{x=-a}+\left.\left(B v_{i}^{-(1)}, v_{j}^{-(1)}\right)\right|_{x=a} \\
& +\left.\left(B v_{i}^{-(1)}, v_{j}^{-(1)}\right)\right|_{x=-a} \\
& +\left.\frac{1}{\beta}\left(\left(A v_{i}^{+(2)}+B v_{i}^{+(1)}\right), v_{j}^{-(1)}\right)\right|_{x=a} \\
& -\left.\frac{1}{\beta}\left(\left(A v_{i}^{+(2)}-B v_{i}^{+(1)}\right), v_{j}^{-(1)}\right)\right|_{x=-a} \\
& +\left.\frac{1}{\beta}\left(\left(A v_{j}^{+(2)}+B v_{j}^{+(1)}\right), v_{i}^{-(1)}\right)\right|_{x=a} \\
& -\left.\frac{1}{\beta}\left(A v_{j}^{+(2)}-B v_{j}^{+(1)}, v_{i}^{-(1)}\right)\right|_{x=-a}+\left.\left(F_{1}, v_{i}^{-(1)}\right)\right|_{x=a}
\end{aligned}
$$

$$
\begin{aligned}
& +\left.\left(F_{1}, v_{i}^{-(1)}\right)\right|_{x=-a}+\left.\left(F_{1}, v_{j}^{-(1)}\right)\right|_{x=a} \\
& \left.+\left.\left(F_{1}, v_{j}^{-(1)}\right)\right|_{x=-a}\right] \\
& \left.-\int_{-a}^{a}\left(\left(A \frac{\partial v_{i}^{(2)}}{\partial x}, v_{j}^{(1)}\right)+\left(A^{\prime} \frac{\partial v_{i}^{(1)}}{\partial x}, v_{j}^{(2)}\right)\right) d x\right\} \\
& =\int_{-a}^{a}\left(J\left(\sum_{j=1}^{m} c_{j m} v_{j}\right), v_{i}\right) d x, \quad i=\overline{1, m}, t \in(0, T]
\end{aligned}
$$

$$
c_{i m}(0)=d_{i m}, \quad i=\overline{1, m}
$$

With help of the above shown arguments we now prove that $r_{m}(t)$ is bounded in some time interval $\left[0, T_{m}\right]$, where $U_{m}(t, x)=r_{m}(t) \omega_{m}(t, x), T_{m} \approx \mathrm{O}\left(\left(\left\|U_{0 m}\right\|-r_{m 1}(0)\right)^{-1}\right), T_{m} \geq$ $T \forall m$, and

$$
\begin{aligned}
& \|\| U_{m}\left\|_{L^{2}[-a, a]}-r_{m 1}\right\|_{C[0, T]} \\
& \quad \leq C_{2}\left(\left\|U_{0}\right\|_{L^{2}[-a, a]}-r_{1}(0)\right),
\end{aligned}
$$

where $C_{2}$ is constant and independent from $m$ and $r_{m 1}(t)$ is a particular solution of Riccati equation about $r_{m}(t)$.

Problem (40)-(41) represents a Cauchy problem for the ordinary system of differential equations. Existence of the solution of problem (40)-(41) follows from theory of ordinary system of differential equations (Picard's existence and uniqueness theorem) [20]. Hence the existence of the solution of problem (34)-(37) follows.

Thus, according to estimation (43) the sequence $\left\{U_{m}-\right.$ $\left.r_{m 1}\right\}$ of approximate solutions of problem (5)-(7) is uniformly bounded in function space $C\left([0, T] ; L^{2}[-a, a]\right)$. Moreover, the homogeneous system of equations $\tau E+(1 / \alpha) A \xi$ with respect to $\tau$, $\xi$ has only a trivial solution. Then it follows from results in [21] that $U_{m}-r_{m 1} \rightarrow U-r_{1}$ is week in $C\left([0, T] ; L^{2}[-a, a]\right)$ and $J\left(U_{m}\right) \rightarrow J(U)$ is week in $C\left([0, T] ; L^{2}[-a, a]\right)$ as $m \rightarrow$ $\infty$. Further it can be shown with standard methods that limit element is a weak solution of problem (5)-(7).

The uniqueness of solution of problem (5)-(7) is proved by contradiction. Let problem (5)-(7) have two different solutions $u_{1}, w_{1}$ and $u_{2}, w_{2}$. We denote them again by $u=u_{1}-u_{2}$ 
and $w=w_{1}-w_{2}$. Then with respect to new values of $u$ and $w$ we obtain the following problem:

$$
\begin{aligned}
\frac{\partial u}{\partial t}+A \frac{\partial w}{\partial x} & =J_{1}\left(u_{1}, w_{1}\right)-J_{1}\left(u_{2}, w_{2}\right) \\
\frac{\partial w}{\partial t}+A^{\prime} \frac{\partial u}{\partial x} & =J_{2}\left(u_{1}, w_{1}\right)-J_{2}\left(u_{2}, w_{2}\right), \\
x \in(-a, a) & \\
\left.u\right|_{t=0} & =0, \\
\left.w\right|_{t=0} & =0, \\
\left.\left(A w^{-}+B u^{-}\right)\right|_{x=-a} & =\left.\frac{1}{\beta}\left(A w^{+}-B u^{+}\right)\right|_{x=-a}, \\
\left.\left(A w^{-}-B u^{-}\right)\right|_{x=a} & =\left.\frac{1}{\beta}\left(A w^{+}+B u^{+}\right)\right|_{x=a} .
\end{aligned}
$$

We prove that solution of problem (44)-(46) is trivial. Hence the uniqueness of the solution of problem (5)-(7) follows.

Using the method which was mentioned before we get (see equality (14))

$$
\begin{aligned}
& \frac{1}{2} \frac{d}{d t} \int_{-a}^{a}[(u, u)+(w, w)] d x+\left.\left(B u^{-}, u^{-}\right)\right|_{x=a} \\
& \quad+\left.\left(B u^{-}, u^{-}\right)\right|_{x=-a}-\left.\frac{1}{\beta}\left(\left(A w^{+}-B u^{+}\right), u^{-}\right)\right|_{x=-a} \\
& \quad+\left.\frac{1}{\beta}\left(\left(A w^{+}+B u^{+}\right), u^{-}\right)\right|_{x=a} \\
& \quad=\int_{-a}^{a}\left[\left(\left(J_{1}\left(u_{1}, w_{1}\right)-J_{1}\left(u_{2}, w_{2}\right)\right), u\right)\right. \\
& \left.\quad+\left(\left(J_{2}\left(u_{1}, w_{1}\right)-J_{2}\left(u_{2}, w_{2}\right)\right), w\right)\right] d x .
\end{aligned}
$$

Let us transform the integrand on the right side of the equality as

$$
\begin{aligned}
& \left(\left(J_{1}\left(u_{1}, w_{1}\right)-J_{1}\left(u_{2}, w_{2}\right)\right), u\right) \\
& =\frac{\sigma_{2}-\sigma_{0}}{2}\left[\left(f_{00,1} f_{02,1}-\frac{f_{01,1}^{2}}{\sqrt{3}}\right)-\left(f_{00,2} f_{02,2}\right.\right. \\
& \left.\left.-\frac{f_{01,2}^{2}}{\sqrt{3}}\right)\right]\left(f_{02,1}-f_{02,2}\right)=\frac{\sigma_{2}-\sigma_{0}}{2}\left[\left(f_{00,1} f_{02,1}\right.\right. \\
& \left.\left.-f_{00,2} f_{02,2}\right)-\frac{1}{\sqrt{3}}\left(f_{01,1}^{2}-f_{01,2}^{2}\right)\right]\left(f_{02,1}-f_{02,2}\right) \\
& =\frac{\sigma_{2}-\sigma_{0}}{2}\left[\left(f_{00,1}-f_{00,2}\right) f_{02,1}+f_{00,2}\left(f_{02,1}\right.\right. \\
& \left.\left.-f_{02,2}\right)-\frac{1}{\sqrt{3}}\left(f_{01,1}-f_{01,2}\right)\left(f_{01,1}+f_{01,2}\right)\right]\left(f_{02,1}\right. \\
& \left.-f_{02,2}\right) ;
\end{aligned}
$$

$$
\begin{aligned}
& \left(\left(J_{2}\left(u_{1}, w_{1}\right)-J_{2}\left(u_{2}, w_{2}\right)\right), u\right)=\left(\left(J_{03}\left(u_{1}, w_{1}\right)\right.\right. \\
& \left.\left.\quad-J_{03}\left(u_{2}, w_{2}\right)\right),\left(f_{03,1}-f_{03,2}\right)\right)+\left(\left(J_{11}\left(u_{1}, w_{1}\right)\right.\right. \\
& \left.\left.-J_{11}\left(u_{2}, w_{2}\right)\right),\left(f_{11,1}-f_{11,2}\right)\right)=\left(\frac { 1 } { 4 } \left(\sigma_{3}+3 \sigma_{1}\right.\right. \\
& \left.-4 \sigma_{0}\right)\left[\left(f_{00,1}-f_{00,2}\right) f_{03,1}+f_{00,2}\left(f_{03,1}-f_{03,2}\right)\right] \\
& +\frac{1}{4 \sqrt{5}}\left(2 \sigma_{1}+\sigma_{0}-3 \sigma_{3}\right)\left[\left(f_{01,1}-f_{01,2}\right) f_{02,1}\right. \\
& \left.\left.+f_{01,2}\left(f_{02,1}-f_{02,2}\right)\right]\right)\left(f_{03,1}-f_{03,2}\right)+\left(\sigma_{1}-\sigma_{0}\right) \\
& +\left(\left(f_{00,1}-f_{00,2}\right) f_{11,1}+f_{00,2}\left(f_{11,1}-f_{11,2}\right)\right. \\
& +0.5 \sqrt{\frac{5}{2}}\left[\left(f_{10,1}-f_{10,2}\right) f_{01,1}\right. \\
& \left.+f_{10,2}\left(f_{01,1}-f_{01,2}\right)\right]-\sqrt{\frac{2}{15}}\left[\left(f_{01,1}-f_{01,2}\right) f_{02,1}\right. \\
& \left.\left.+f_{01,2}\left(f_{02,1}-f_{02,2}\right)\right]\right)\left(f_{11,1}-f_{11,2}\right) .
\end{aligned}
$$

Once again we use spherical representation of $u=r(t) w_{1}(t$, $x)$ and $w=r(t) w_{2}(t, x)$, where $r(t)=\|U(t, \cdot)\|_{L^{2}[-a, a]}$. Then concerning $r(t)$ we obtain new initial value problem

$$
\begin{aligned}
\frac{d r}{d t}+r P(t) & =r^{2} Q_{1}(t), \\
r(0) & =0,
\end{aligned}
$$

where $P(t)$ has the same value as in (15) and

$$
\begin{aligned}
& Q_{1}(t)=\frac{\sigma_{2}-\sigma_{0}}{2} \int_{-a}^{a}\left[\left(\omega_{00,1}-\omega_{00,2}\right) \omega_{02,1}\right. \\
& +\omega_{00,2}\left(\omega_{02,1}-\omega_{02,2}\right)-\frac{1}{\sqrt{3}}\left(\omega_{01,1}-\omega_{01,2}\right)\left(\omega_{01,1}\right. \\
& \left.\left.+\omega_{01,2}\right)\right]\left(\omega_{02,1}-\omega_{02,2}\right) d x+\frac{1}{4}\left(\sigma_{3}+3 \sigma_{1}\right. \\
& \left.\quad-4 \sigma_{0}\right) \int_{-a}^{a}\left[\left(\omega_{00,1}-\omega_{00,2}\right) \omega_{03,1}++\omega_{00,2}\left(\omega_{03,1}\right.\right. \\
& \left.\left.\quad-\omega_{03,2}\right)\right]\left(\omega_{03,1}-\omega_{03,2}\right) d x+\frac{1}{4 \sqrt{5}}\left(2 \sigma_{1}+\sigma_{0}-3 \sigma_{3}\right) \\
& \cdot \int_{-a}^{a}\left[\left(\omega_{01,1}-\omega_{01,2}\right) \omega_{02,1}+\omega_{01,2}\left(\omega_{02,1}-\omega_{02,2}\right)\right] \\
& \cdot\left(\omega_{03,1}-\omega_{03,2}\right) d x+\left(\sigma_{1}-\sigma_{0}\right) \int_{-a}^{a}\left[\left(\omega_{00,1}\right.\right. \\
& \left.\quad-\omega_{00,2}\right) \omega_{11,1}+\omega_{00,2}\left(\omega_{11,1}-\omega_{11,2}\right)+\frac{1}{2}
\end{aligned}
$$




$$
\begin{aligned}
& \cdot \sqrt{\frac{5}{2}}\left[\left(\omega_{10,1}-\omega_{10,2}\right) \omega_{01,1}+\omega_{10,2}\left(\omega_{01,1}-\omega_{01,2}\right)\right] \\
& -\sqrt{\frac{2}{15}}\left[\left(\omega_{01,1}-\omega_{01,2}\right) \omega_{02,1}+\omega_{01,2}\left(\omega_{02,1}-\omega_{02,2}\right)\right] \\
& \left.\cdot\left(\omega_{11,1}-\omega_{11,2}\right)\right] d x .
\end{aligned}
$$

The general solution of (49) is

$$
\begin{aligned}
r(t) & =\exp \left(-\int_{0}^{t} P(\tau) d \tau\right) \\
\cdot & {\left[C-\int_{0}^{t} Q_{1}(\tau) \exp \left(-\int_{0}^{\tau} P(\xi) d \xi\right) d \tau\right]^{-1} . }
\end{aligned}
$$

Solution of (49), which is satisfying homogeneous condition (50), is trivial; that is, $r(t)=0$. Hence, $\|U\|_{C\left([0, T] ; L^{2}[-a, a]\right)}=0$ and $u_{1}=u_{2}$ and $w_{1}=w_{2}$.

The theorem is proved.

\section{Competing Interests}

The authors declare that they have no competing interests.

\section{References}

[1] H. Grad, "On the kinetic theory of rarefied gases," Communications on Pure and Applied Mathematics, vol. 2, no. 4, pp. 331-407, 1949.

[2] H. Grad, "Principle of the kinetic theory of gases," in Thermodynamics of Gases, vol. 12 of Handbuch der Physik, pp. 205-294, Springer, Berlin, Germany, 1958.

[3] A. Sakabekov, Initial-Boundary Value Problems for the Boltzmann's Moment System Equations Gylym, Almaty, 2002.

[4] C. Cercignani, Theory and Application of the Boltzmann Equation, Instituto di Matematica, Milano, Italy, 1975.

[5] M. N. Kogan, Dynamic of Rarefied Gas, Nauka, Moscow, Russia, 1967.

[6] K. Kumar, "Polynomial expansions in kinetic theory of gases," Annals of Physics, vol. 37, no. 1, pp. 113-141, 1966.

[7] A. Sakabekov and Y. Auzhani, "Boundary conditions for the onedimensional nonlinear nonstationary Boltzmann's moment system equations," Journal of Mathematical Physics, vol. 55, Article ID 123507, 2014.

[8] C. D. Levermore, "Moment closure hierarchies for kinetic theories," Journal of Statistical Physics, vol. 83, no. 5-6, pp. 10211065, 1996.

[9] G. Mascali and V. Romano, "A hydrodynamical model for holes in silicon semiconductors: the case of non-parabolic warped bands," Mathematical and Computer Modelling, vol. 53, no. 1-2, pp. 213-229, 2011.

[10] G. Mascali and V. Romano, "A non parabolic hydrodynamical subband model for semiconductors based on the maximum entropy principle," Mathematical and Computer Modelling, vol. 55, no. 3-4, pp. 1003-1020, 2012.

[11] V. D. Camiola and V. Romano, "2DEG-3DEG charge transport model for MOSFET based on the maximum entropy principle,"
SIAM Journal on Applied Mathematics, vol. 73, no. 4, pp. 14391459, 2013.

[12] G. Alì, G. Mascali, V. Romano, and R. C. Torcasio, "A hydrodynamical model for covalent semiconductors with a generalized energy dispersion relation," European Journal of Applied Mathematics, vol. 25, no. 2, pp. 255-276, 2014.

[13] V. D. Camiola and V. Romano, "Hydrodynamical model for charge transport in graphene," Journal of Statistical Physics, vol. 157, no. 6, pp. 1114-1137, 2014.

[14] S. Mischler, "Kinetic equations with Maxwell boundary conditions," Annales Scientifiques de l'Ecole Normale Superieure, vol. 43, no. 5, pp. 719-760, 2010.

[15] V. G. Neudachin and U. F. Smirnov, Nucleon Association of Easy Kernel, Nauka, Moscow, Russia, 1969.

[16] M. Moshinsky, The Harmonic Oscillator in Modern Physics: from Atoms to Quarks, 1960.

[17] S. I. Pokhozhaev, "On an approach to nonlinear equation," Doklady Akademii Nauk SSSR, vol. 247, pp. 1327-1331, 1979.

[18] E. Kamke, Differentialgleichungen Lösungsmethoden und Lösungen, I. Gewohuliche Differentialgleichungen, B.G.Teubner, Leipzig, Germany, 1977.

[19] A. Tungatarov and D. K. Akhmed-Zaki, "Cauchy problem for one class of ordinary differential equations," International Journal of Mathematical Analysis, vol. 6, no. 14, pp. 695-699, 2012.

[20] M. Tenenbaum and H. Pollard, Ordinary Differential Equations, Harper and Row, New York, NY, USA, 1963.

[21] L. Tartar, "Compensated compactness and applications to partial differential equations," in Proceedings of the Non-Linear Analysis and Mechanics, Heriot-Watt Symposium, R. J. Knops, Ed., vol. 4 of Research Notes in Math, pp. 136-212, 1979. 


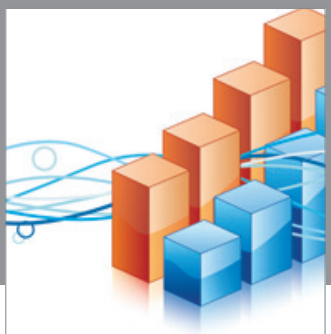

Advances in

Operations Research

vatem alat4

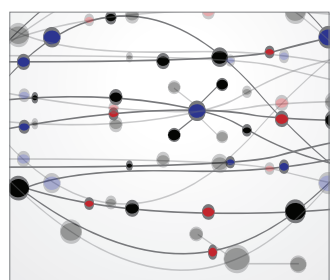

\section{The Scientific} World Journal
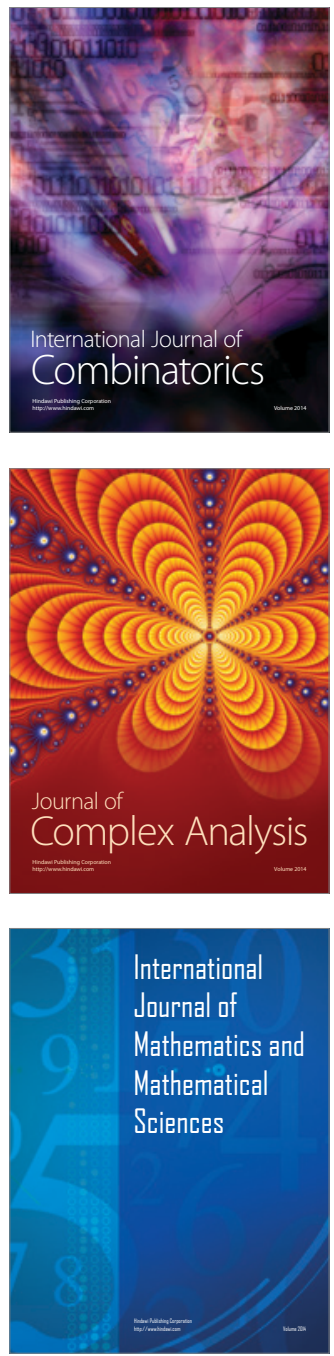
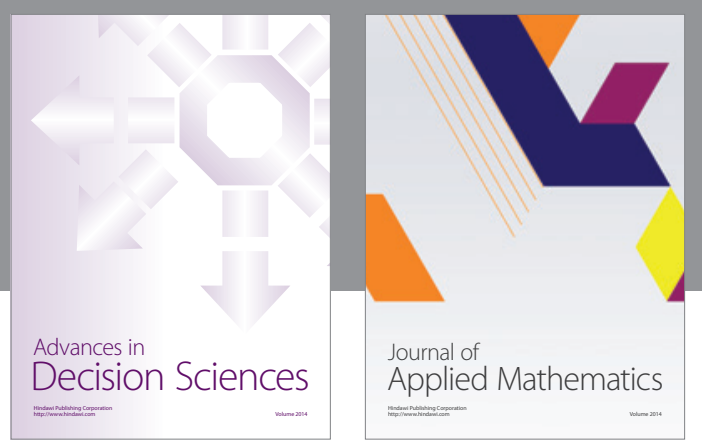

Algebra

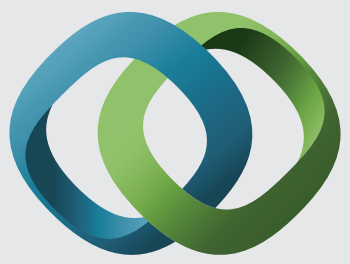

\section{Hindawi}

Submit your manuscripts at

http://www.hindawi.com
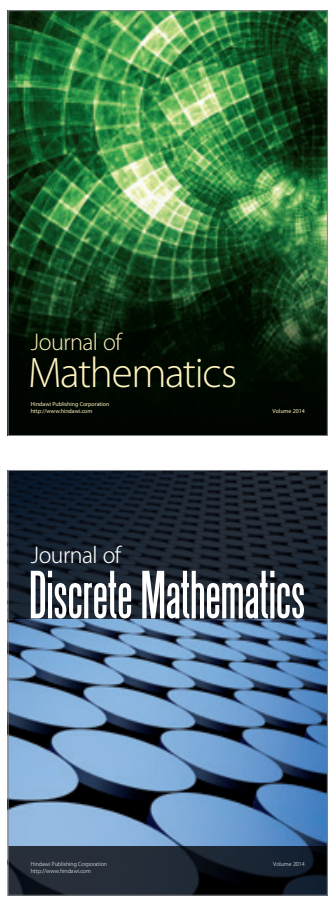

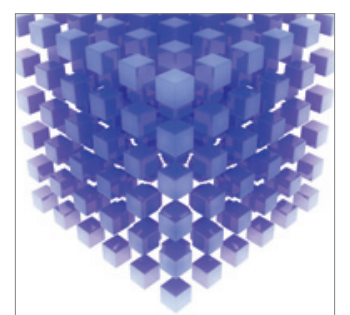

Mathematical Problems in Engineering
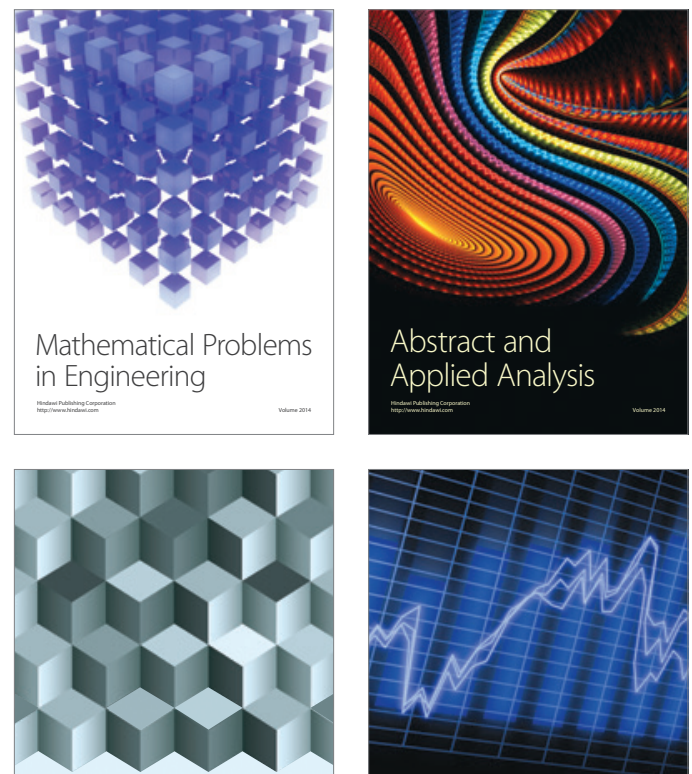

Journal of

Function Spaces

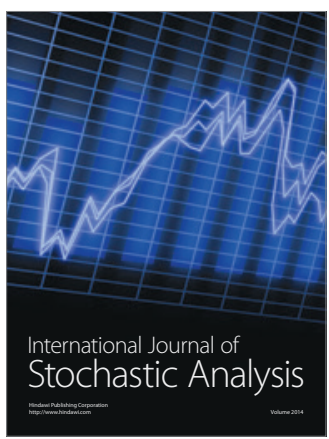

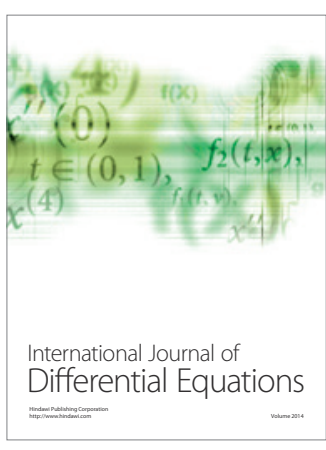
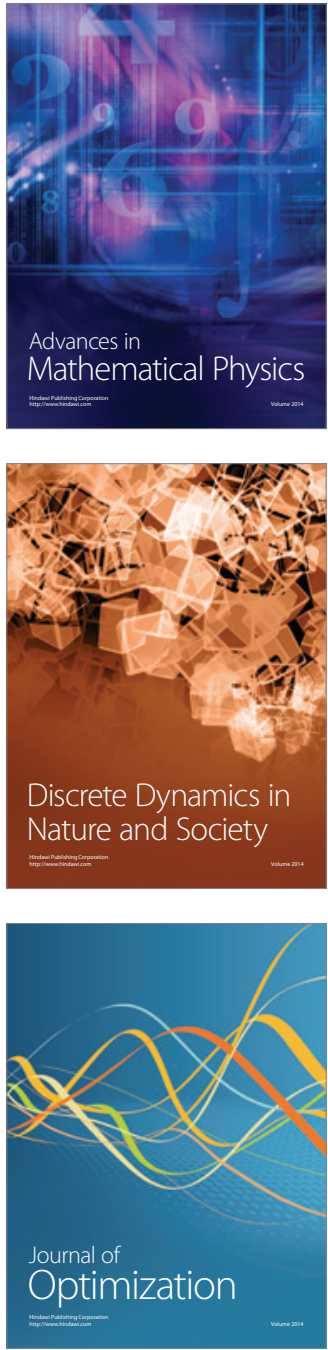